\title{
SI(100) VERSUS GE(100): WATCHING THE INTERFACE FORMATION FOR THE GROWTH OF III-V-BASED SOLAR CELLS ON ABUNDANT SUBSTRATES
}

\author{
S. Brückner ${ }^{1}$, O. Supplie ${ }^{1}$, E. Barrigón ${ }^{1,2}$, P. Kleinschmidt ${ }^{1}$, A. Dobrich ${ }^{1}$, I. Rey-Stolle ${ }^{2}$, C. Algora ${ }^{2}$, H. Döscher ${ }^{1}$, \\ T. Hannappel ${ }^{1}$ \\ ${ }^{1}$ Helmholtz Center Berlin for Materials and Energy, Hahn-Meitner-Platz 1, Berlin, Germany \\ ${ }^{2}$ Instituto de Energía Solar, Universidad Politécnica de Madrid, Spain
}

\begin{abstract}
We investigated the atomic surface properties of differently prepared silicon and germanium (100) surfaces during metal-organic vapour phase epitaxy/chemical vapour deposition (MOVPE/MOCVD), in particular the impact of the MOVPE ambient, and applied reflectance anisotropy/difference spectroscopy (RAS/RDS) in our MOVPE reactor to in-situ watch and control the preparation on the atomic length scale for subsequent III-V-nucleation. The technological interest in the predominant opto-electronic properties of III-Vcompounds drives the research for their heteroepitaxial integration on more abundant and cheaper standard substrates such as $\mathrm{Si}(100)$ or $\mathrm{Ge}(100)$. In these cases, a general task must be accomplished successfully, i.e. the growth of polar materials on nonpolar substrates and, beyond that, very specific variations such as the individual interface formation and the atomic step structure, have to be controlled. Above all, the method of choice to grow industrial relevant high-performance device structures is MOVPE, not normally compatible with surface and interface sensitive characterization tools, which are commonly based on ultrahigh vacuum (UHV) ambients. A dedicated sample transfer system from MOVPE environment to UHV enabled us to benchmark the optical in-situ spectra with results from various surfaces science instruments without considering disruptive contaminants. X-ray photoelectron spectroscopy (XPS) provided direct observation of different terminations such as arsenic and phosphorous and verified oxide removal under various specific process parameters. Absorption lines in Fourier-transform infrared (FTIR) spectra were used to identify specific stretch modes of coupled hydrides and the polarization dependence of the anti-symmetric stretch modes distinguished different dimer orientations. Scanning tunnelling microscopy (STM) studied the atomic arrangement of dimers and steps and tip-induced $\mathrm{H}$-desorption proved the saturation of dangling bonds after preparation. In-situ RAS was employed to display details transiently such as the presence of $\mathrm{H}$ on the surface at lower temperatures ( $T$ $<800^{\circ} \mathrm{C}$ ) and the absence of $\mathrm{Si}-\mathrm{H}$ bonds at elevated annealing temperature and also surface terminations. $\mathrm{Ge}$ buffer growth by the use of $\mathrm{GeH}_{4}$ enables the preparation of smooth surfaces and leads to a more pronounced amplitude of the features in the spectra which indicates improvements of the surface quality.
\end{abstract}

\section{INTRODUCTION}

The handling of germanium based high-performance devices shows impressively that the common task, i.e. growing successfully polar III-V material on non-polar templates, can work out rather perfectly, even though the microscopic III-V/Ge(100) nucleation and its interface formation has not been described in detail so far. Triple junction solar cells with ultimate performance, which are today's most efficient photovoltaic devices with conversion efficiencies exceeding $40 \%$, can be grown with III-V compound semiconductors on $\mathrm{Ge}(100)$ substrates employing MOVPE. A great landmark for solar cell-relevant breakthrough technologies is the merge of silicon and III-V technologies. This notorious old problem is still not solved, and even though a lot of studies of $\mathrm{Si}$ surfaces prepared in ultrahigh-vacuum (UHV) have been published in the past, the challenge is the growth of III-V compounds on $\mathrm{Si}(100)$ surfaces without significantly deteriorating the opto-electronic properties of the III-V material. It is getting even more difficult, when regarding device structures prepared in MOVPE ambients, as the knowledge for these interfaces is much less.

In our work, the above mentioned topics have been tackled and the delicate MOVPE preparation of critical interfaces was monitored on the atomic scale with optical in-situ spectroscopy and, associated with these signals, sophisticated analytic tools such as low energy electron diffraction (LEED), STM, FTIR, and XPS. Employing these UHV-based tools, the optical in-situ signals have been directly benchmarked with regard to the atomic surface structure, the chemical bonds, the symmetry of the surface reconstructions, and the chemical ingredients on the surface, respectively. Via this experimental strategy, the MOVPE-growth of silicon and germanium and its surface preparation have been studied in detail and approaches of growing III-Vs on $\mathrm{Ge}(100)$ have been compared to those of nucleating III-Vs on $\mathrm{Si}(100)$.

Subsequent III-V deposition requires the complete removal of intrinsic oxides $[1,2]$ and the generation of a suitable atomic surface structure. The reduction of defects, impurities and undesirable anti-phase disorder necessitates the formation of well-defined step structures such as double layer steps instead of the typical two-domain $(2 \times 1) /(1 \times 2)$ surface reconstruction of $\mathrm{Si}(100)$ associated with single layer steps [3]. 


\section{EXPERIMENTAL}

Our investigations of the $\mathrm{Si}$ and $\mathrm{Ge}(100)$ surfaces were performed on substrates with a misorientation of $6^{\circ}$ towards the [011] direction. The preparation was carried out in a commercially available MOVPE reactor (Aixtron AIX200) especially modified for surface characterization. An optical view port at the reactor allowed in situ monitoring of the sample surface by RAS (LayTec EpiRAS 200). A dedicated sample transfer system involving a mobile UHV chamber enabled contamination-free access to several surface analysis systems such as LEED (Specs ErLEED 100-A), STM (Specs Aarhus 150), or XPS (Specs Focus 500 and Phoibos 100).
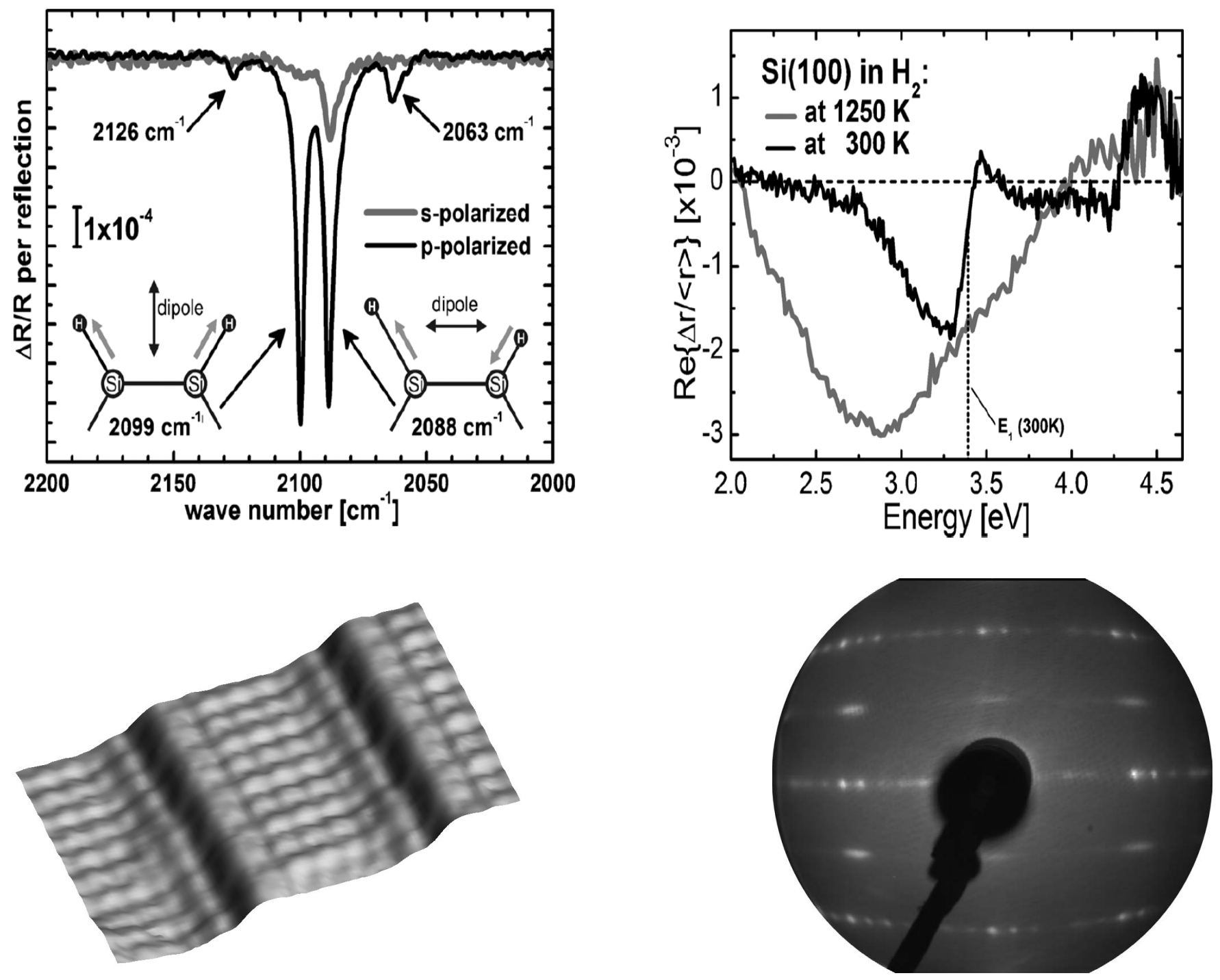

Figure 1 - FTIR spectroscopy, in-situ RAS, and STM images of Si(100) surfaces $\left(2^{\circ}\right.$ miscut towards [111]) prepared via MOVPE; showing the presence/absence of hydrogen at different annealing temperatures and the corresponding atomic step structure of the Si(100) surfaces. 


\section{RESULTS}

In our studies, XPS provided direct observation of the oxide removal under various specific process parameters (see [1], Fig. 2).

Our RA spectra of $\mathrm{Si}(100)$ and $\mathrm{Ge}(100)$ substrates (Figs. 1, 2) have been measured with different miscuts and terminations in the MOVPE environment and were consistent with results reported in the literature that were derived from UHV studies. Benchmarking of the RAS signals enabled us to observe the reconstruction $[3,4]$ and the atomic structure of the surfaces in-situ, also by transient RAS measurements [3]. On $\mathrm{Si}(100)$, coupled $\mathrm{Si}-\mathrm{H}$ monohydrides [5] were identified as stretch modes via specific absorption lines in Fouriertransform infrared spectra, in agreement with Sidimers observed by scanning tunneling microscopy (STM). The polarization dependence of the antisymmetric stretch modes distinguished different dimer orientations and verified a clear preference for one of the $(2 \times 1) /(1 \times 2)$ surface reconstruction domains. Tipinduced $\mathrm{H}$-desorption proved the complete saturation of dangling bonds after VPE-preparation. In-situ RAS showed the deoxidation of $\mathrm{Si}(100)$ and the absence/presence of $\mathrm{H}$ on the surface. Compared to $\mathrm{Ge}(100)$, where abrupt deoxidation is observed with RAS at low temperatures $\left(T>450^{\circ} \mathrm{C}\right)$, the deoxidation requires high temperatures $\left(T>950^{\circ} \mathrm{C}\right)$ for $\mathrm{Si}(100)$. While the $\mathrm{Si}(100)$ surface is free of hydrogen at higher temperatures $\left(\mathrm{T}>800^{\circ} \mathrm{C}\right), \mathrm{H}$ adsorbs during cooling down in $\mathrm{H}_{2}$ according to RAS (Fig.1). For $\mathrm{Si}(100)$ surfaces with $6^{\circ}$ miscut orientation, STM images of Fig.1 (bottom panel, left hand side) reveal a double step configuration, so called $D_{B}$ double steps [6]. These are also reflected by the LEED pattern shown in Fig. 1 (bottom panel, right hand side), which displays a preferentially one-domain, (2x1)reconstructed surface.

In Fig. 2, XP spectra in the range of the O1s, C1s and $\mathrm{Ge} 2 \mathrm{p} 3 / 2$ photoemission lines of the epiready $\mathrm{Ge}(100)$ substrates (black line) and the substrate surface after $\mathrm{H}_{2}$ annealing in MOVPE (green line) are depicted. On the epiready substrate the $01 \mathrm{~s}$ binding energy observed at about $532.4 \mathrm{eV}$ complies well with the chemical shift of $+1.4 \mathrm{eV}$ compared to the elemental O1s line at $531 \mathrm{eV}$, as expected for oxygen bound in $\mathrm{GeO}_{2}$ [7]. After $\mathrm{H}_{2}$ annealing two small peaks at 534 and $525 \mathrm{eV}$ are measured in this range that can be assigned to the Auger lines of clean $\mathrm{Ge}, \mathrm{L}_{3} \mathrm{M}_{23} \mathrm{M}_{23}$ and $\mathrm{L}_{2} \mathrm{M}_{23} \mathrm{M}_{23}$, respectively [8]. At the position of the $\mathrm{Ge} 2 \mathrm{p}_{3 / 2}$ line we observed a peak at $1217.5 \mathrm{eV}$, associated to elemental $\mathrm{Ge}$ [7], and the Ge2p photoemission from the $\mathrm{GeO}_{2}$ layer formed an additional peak structure at about $1220.5 \mathrm{eV}$. After hydrogen annealing the peaks associated to $\mathrm{GeO}_{x}$ are vanished and the peak intensities related to elemental $\mathrm{Ge}$ are increased. No peak intensity was measured for the $\mathrm{C}$ 1s photoemission line after annealing in $\mathrm{H}_{2}$ at $700{ }^{\circ} \mathrm{C}$. XPS analysis of the homoepitaxially prepared samples also confirmed the absence of oxygen or any other contamination on the surface after growth.

Fig. 2 also shows the corresponding in situ RA spectra of vicinal $\mathrm{Ge}(100)$ measured at $20^{\circ} \mathrm{C}$ during differen $\mathrm{t}$ stages of the preparation in the MOVPE reactor: The oxidized epiready substrate (black line), the clean surface after deoxidation (green line) and after homoepitaxy (red line), all cooled down in a $\mathrm{H}_{2}$ MOVPE-ambient. The oxidized surface exhibits a featureless spectrum due to the amorphous oxide layer. The spectrum of the clean deoxidized surface (green line) evolves during the hydrogen annealing for temperatures $>450^{\circ} \mathrm{C}$. The spectrum shows local maxima around the critical point energies of $\mathrm{Ge}(100)$ $E_{1}$ and $E_{1}+\Delta_{1}$ around $2.2 \mathrm{eV}$ and $E_{2}$ at $4.3 \mathrm{eV}$, and also two negative features, a narrow structure at 1.9 $\mathrm{eV}$ and a broad structure around $3 \mathrm{eV}$. The spectrum agrees very well to spectra of clean vicinal $\mathrm{Ge}(100)$ prepared in UHV [9], [10]. For $\mathrm{Si}(100)$ it was shown in [3] that the surface is terminated with monohydrides after processing in a $\mathrm{H}_{2}$ MOVPE-ambient, and it exhibits the characteristic RA spectrum related to a monohydride termination. The measured RA spectrum of the $\mathrm{Ge}(100)$ surface corresponds to the spectrum of the $\mathrm{H}$-free surface. However, so far it is not clear if the $\mathrm{Ge}(100)$ surface is $\mathrm{H}$ terminated after $\mathrm{H}_{2}$ annealing or not, and if the RAS of vicinal $\mathrm{Ge}(100)$ is sensitive to hydrogen adsorption as it is for $\mathrm{Si}(100)$.

The homoepitaxially prepared surface displays a spectrum with the same lineshape but all features are much more pronounced (red line). According to RAS studies of other semiconductor surfaces this indicates a improved atomic order on the surface. Witkowski et al. [11] showed in a detailed RAS and STM study of the clean vicinal $\mathrm{Si}(100)$ surface that the intensity of the RA spectra is very sensitive to deteriorations and can be used to measure the domain ratio on the surface. For III-V surfaces RAS also is highly sensitive to atomic order on the surface as shown for example for the $\operatorname{InP}(100)$ [12] and $\mathrm{GaP}(100)$ surfaces [13].

We also applied LEED to analyze the surface quality and domain ratio of the samples (Fig. 2, lower panel). The corresponding LEED images of the $6^{\circ}$ offcut $\mathrm{Ge}(100)$ samples after deoxidation (Fig. 2, lower panel, green) and $\mathrm{Ge}$ buffer growth (Fig. 2. lower panel, red) taken at $168 \mathrm{eV}$ show pronounced spot splitting along the [011] direction resulting from step edges at terraces of regular width. Half-ordered spots parallel to [0-11] originate in the $(2 \times 1)$-like dimerized surface reconstruction and indicate a single-domain respectively highly preferential domain surface. In comparison, the LEED images do not reveal any differences in surface quality regarding domain ratio or spot intensities. First STM images of the deoxidized surface (not shown here) confirmed the presence of mainly $D_{B}$ type double layer steps. 

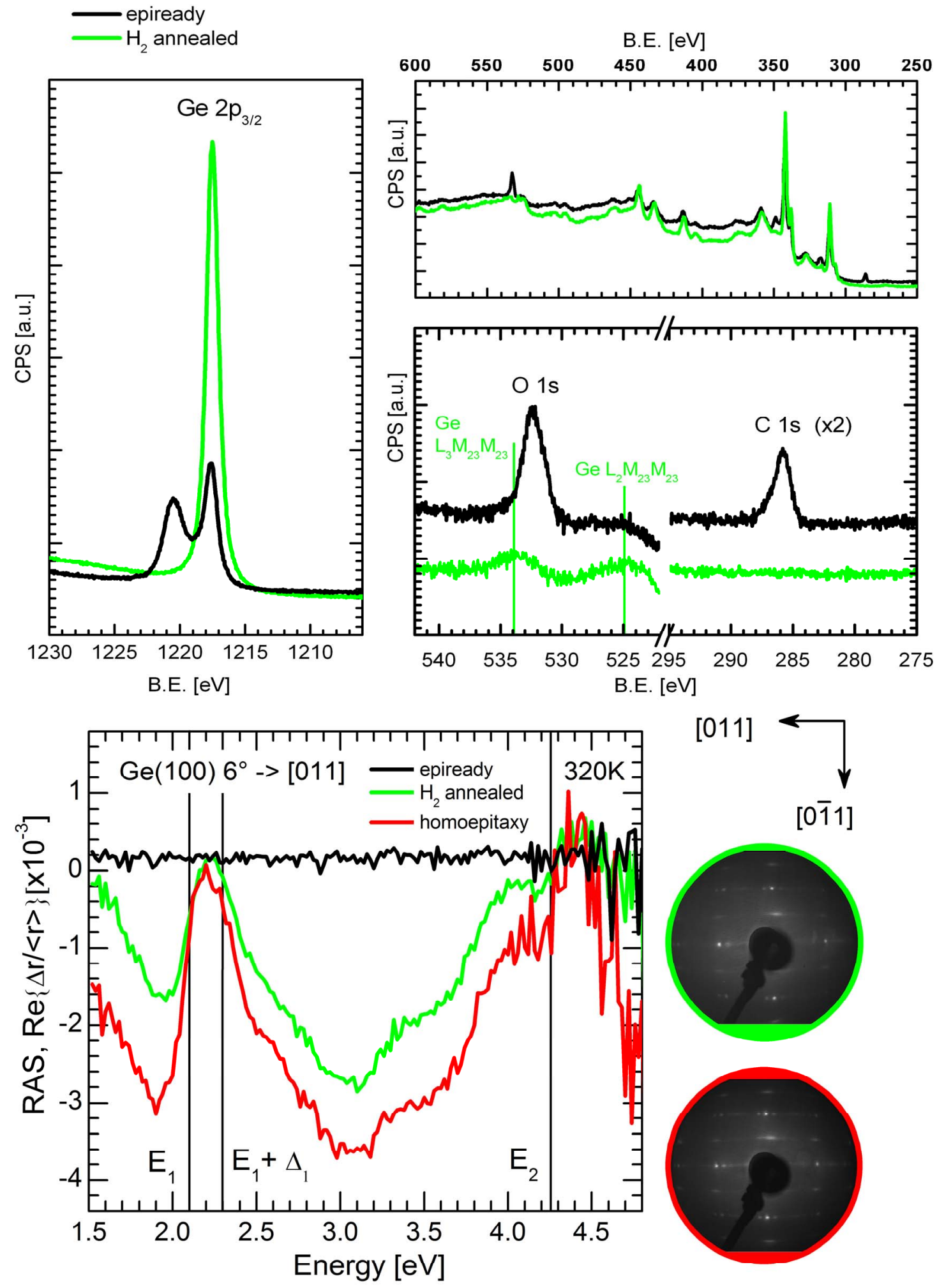

Fig.2:

Upper panel: XP spectra in the range of the 01s, C1s and Ge2p3/2 photoemission lines of the epiready $\mathrm{Ge}(100)$ substrates (black line) and the substrate surface after $\mathrm{H}_{2}$ annealing at $700^{\circ} \mathrm{C}$ in MOVPE (green line). Lower panel: Corresponding RA spectra (black vs. green curves) and LEED images. The red curve shows an RA spectrum of a $\mathrm{Ge}(100)$ surface after homoepitaxial growth. 


\section{CONCLUSION}

The atomic surface properties of differently prepared silicon and germanium (100) surfaces during MOVPE preparation were investigated. Surface reconstruction, deoxidation, $\mathrm{H}$-termination, domain order, degree of the atomic order, and, in particular, the impact of the MOVPE ambient were determined by RAS in our MOVPE reactor to in-situ watch and control the preparation on the atomic length scale for subsequent III-V-nucleation. RAS was used for in situ observation of oxide removal from vicinal $\mathrm{Si}$ and $\mathrm{Ge}$ (100) substrates. Ge buffer growth by the use of $\mathrm{GeH}_{4}$ enables the preparation of smooth surfaces and leads to a more pronounced amplitude of the features in the spectra which indicates improvements of the surface quality. LEED images confirmed a preferential $(2 \times 1)$ surface reconstruction for both the deoxidized and the homoepitaxially prepared $\mathrm{Ge}(100)$ surface. Benchmarking of the spectra to other surface sensitive techniques like ultraviolet photoelectron spectroscopy (UPS) or STM might lead to a better understanding of the observed differences. In future work the influences of $\mathrm{Ge}$ buffer growth on the subsequent III-V heteroepitaxy will be studied. We have shown that homoepitaxial growth with $\mathrm{SiH}_{4}$ and $\mathrm{GeH}_{4}$, respectively, enhances the surface quality which can be further improved by appropriate annealing conditions as shown by RAS and LEED.

\section{ACHNOWLEDGEMENTS}

This work was supported by the BMBF (Project no. 03SF0329C).

\section{REFERENCES}

[1] H. Döscher, S. Brückner, A. Dobrich, C. Höhn, P. Kleinschmidt, T. Hannappel, J. Cryst. Growth 315 (2010) 10

[2] H. Döscher, S. Brückner, T. Hannappel, Journal of Crystal Growth 318 (2011) 563

[3] H.Döscher, A. Dobrich, S. Brückner, P. Kleinschmidt, T. Hannappel, Appl. Phys. Lett. 97, 151905 (2010)

[4] H. Döscher, P. Kleinschmidt, T Hannappel, Appl. Surf. Sci. 257 (2010) 574

[5] S. Brückner, H. Döscher, P, Kleinschmidt, T. Hannappel, Appl. Phys. Lett. 98 (2011) 211909

[6] D. J. Chadi, Phys. Rev. Lett. 59, 1691 (1987)

[7] J. Oh, J. C. Campbell, J. Electr. Mat. 33 (2004) 364

[8] E. Antonides, E. C. Janse, G. A. Sawatzky, Phys. Rev. B 15 (1977) 1669

[9] T. Yasuda, L. Mantese, U. Rossow, D. E. Aspnes, Phys. Rev. Lett. 74 (1995) 3431
[10] U. Rossow, L. Mantese, D. E. Aspnes, J. Vac. Sci. Techn. B 18 (2000) 2229

[11] N. Witkowski, R. Coustel, O. Pluchery, Y. Borensztein, Surf. Sci. 600 (2006) 5142

[12] T. Letzig, H.-J. Schimper, T. Hannappel, F. Willig, Phys. Rev. B 71 (2005) 033308

[13] H. Doscher T. Hannappel, J. Appl. Phys. 107 (2010) 123523 